\title{
WHOLE EARTH TELESCOPE OBSERVATIONS OF V471 TAURI: THE NATURE OF THE WHITE DWARF VARIATIONS
}

\author{
J. C. Clemens, ${ }^{1,2}$ R. E. Nather, ${ }^{1}$ D. E. Winget, ${ }^{1,2}$ E. L. Robinson, ${ }^{1}$ M. A. Wood, ${ }^{1,3}$ C. F. Claver, ${ }^{1}$ \\ J. Provencal, ${ }^{1}$ S. J. Kleinman, ${ }^{1}$ P. A. Bradley, ${ }^{1}$ M. L. Frueh, ${ }^{1}$ A. D. Grauer,${ }^{4,5}$ B. P. Hine, ${ }^{6}$ \\ G. Fontaine, ${ }^{2,7,8}$ N. Achilleos, ${ }^{9}$ D. T. Wickramasinghe, ${ }^{9}$ T. M. K. MaraR, ${ }^{10}$ S. Seetha, ${ }^{10}$ \\ B. N. AShoka, ${ }^{10}$ D. O'Donoghue ${ }^{11}$ B. Warner, ${ }^{11}$ D. W. KuRTZ, ${ }^{11}$ P. Martinez, ${ }^{11}$ \\ G. Vauclair, ${ }^{12}$ M. CheVreton, ${ }^{13}$ M. A. Barstow, ${ }^{14}$ A. KanaAn, ${ }^{15}$ S. O. KePler, ${ }^{15}$ \\ T. Augusteisn, ${ }^{16}$ J. van Paradiss, ${ }^{17}$ AND C. J. Hansen ${ }^{18}$ \\ Received 1991 August 12; accepted 1991 December 10
}

\begin{abstract}
We have conducted time-series photometric observations of the binary star V471 Tauri using the Whole Earth Telescope observing network. Our purpose was to determine the mechanism responsible for causing the 555 and $277 \mathrm{~s}$ periodic luminosity variations exhibited by the white dwarf in this binary. Previous observers have proposed that either $g$-mode pulsations or rotation of an accreting magnetic white dwarf could cause the variations, but were unable to decide which was the correct model. Our observations have answered this question.

Learning the cause of the white dwarf variations has been possible because of our discovery of a periodic signal at $562 \mathrm{~s}$ in the Johnson $U$-band flux of the binary. By identifying this signal as reprocessed radiation and using its phase to infer the phase of the shorter wavelength radiation which produces it, we have been able to compare the phase of the $555 \mathrm{~s} U$-band variations to the phase of the X-ray variations. We have found that $U$-band maximum coincides with X-ray minimum. From this result we have concluded that the magnetic rotator model accurately describes the variations we observe, but that models involving $g$-mode pulsations do not. Subject headings: stars: individual (V471 Tauri) - stars: oscillations - white dwarfs
\end{abstract}

\section{INTRODUCTION}

V471 Tau is an eclipsing binary star in the Hyades cluster consisting of a DA white dwarf primary and a $\mathrm{K} 2 \mathrm{~V}$ secondary

${ }^{1}$ Department of Astronomy and McDonald Observatory, University of Texas, Austin, TX 78712.

2 Visiting Astronomer, Canada-France-Hawaii Telescope, operated by the National Research Council of Canada, Centre National de la Recherche Scientifique de France, and the University of Hawaii.

${ }^{3}$ Current postal address: Department of Physics and Space Sciences, Florida Institute of Technology, 150 West University Boulevard, Melbourne, FL 32901.

${ }_{4}$ Department of Physics and Astronomy, University of Arkansas at Little Rock, 2801 South University Avenue, Little Rock, AR 72204.

5 Visiting Observer, $61 \mathrm{~cm}$ telescope at Mauna Kea Observatory, Institute for Astronomy, University of Hawaii.

${ }^{6}$ NASA/Ames Research Center, M.S. 244-4, Moffett Field, CA 94035.

${ }^{7}$ Killam Fellow.

${ }^{8}$ Departement de Physique, Université de Montréal, C.P. 6128, Succ. A., Montréal, PQ Canada, H3C 3J7.

${ }^{9}$ Department of Mathematics, Australian National University, Canberra, Australia.

${ }^{10}$ Indian Space Research Organization, Technical Physics Division, ISRO Satellite Centre, Airport Road, Bangalore 560 017, India.

${ }^{11}$ Department of Astronomy, University of Cape Town, Rondebosch 7700, Cape Province, South Africa.

12 Observatoire Midi-Pyrenees, 14 Avenue E. Belin, 31400 Toulouse, France.

${ }_{13}$ Observatoire de Paris-Meudon, F-92195 Meudon Principal Cedex, France.

${ }_{14}$ Department of Physics and Astronomy, University of Leicester, Leicester LE1 7RH, United Kingdom.

15 Instituto de Fisica, Universidade Federal do Rio Grande do Sul, 91500 Porto Alegre-RS, Brasil.

${ }^{16}$ European Southern Observatory, La Silla, Chile.

17 Universiteit van Amsterdam, Faculteit der Wiskunde en Natuurwetenschappen, Roetersstreat 15,1018 WB, Amsterdam, The Netherlands.

18 J.I.L.A., University of Colorado, Box 440, Boulder, CO 80309. in close orbit, $P_{\text {orb }}=0.521$ days (Nelson \& Young 1970). Because of its likely history as a common-envelope binary and its probable future as a cataclysmic variable, V471 Tau has been the object of much scrutiny. For reviews of the characteristics of this system see Bois, Lanning, \& Mochnacki (1988) and Skillman \& Patterson (1988).

In 1986, Jensen et al. reported their discovery of periodic modulations at 555 and $277 \mathrm{~s}$ in the soft X-ray flux from the white dwarf in V471 Tau. Subsequently, Robinson, Clemens, \& Hine (1988) detected the optical counterparts to the $555 \mathrm{~s}$ oscillations and Winget \& Claver (1989) found the optical counterparts to those at $277 \mathrm{~s}$. Jensen et al. presented two possible models for the system: either the periodic signals are caused by $g$-mode pulsations of the white dwarf, whose temperature places it in a possible instability strip (Winget 1981), or by inhomogeneities in the photosphere of the white dwarf which move in and out of view as the white dwarf rotates.

In the rotation model, the inhomogeneities could be caused by accretion of metal-rich material from the $\mathrm{K}$ star onto the magnetic poles of the white dwarf. Whether the poles are brightened or darkened at X-ray wavelengths depends on the accretion rate. A high accretion rate could cause shock heating near the magnetic poles, brightening them in the X-ray, while a low accretion rate could block the X-rays near the polar regions by depositing metal-rich material onto the white dwarf. Robinson et al. (1988) argued against magnetic poles which are brightened in X-ray, because the required accretion rate of $\sim 10^{-11.5} M_{\odot} \mathrm{yr}^{-1}$ (Jensen et al. 1986) should cause other observable effects which have not been found. They also pointed out that while the deposition of metal-rich material onto regions of the white dwarf might darken those regions at $\mathrm{X}$-ray wavelengths, it would not do so at optical wavelengths; 
in fact, the accretion poles might be optically brightened since the blocked flux from thermal X-rays could be radiated away at longer wavelengths.

Neither Jensen et al. (1986) nor Robinson et al. (1988) were able to distinguish between the rotation and pulsation models on the basis of their data. Since the solution of this problem yields either a new class of pulsating white dwarfs or a new type of interacting binary, we decided to observe V471 Tau with the Whole Earth Telescope (WET) observing network (Nather et al. 1990). In this paper we report the results of those observations.

\section{OBSERVATIONS}

In 1988 November 5-21, we observed V471 Tau with a network of telescopes at seven different observatories, and later obtained follow-up observations with the Canada-FranceHawaii Telescope (CFHT) in Hawaii on December 1-3. Table 1 is a journal of the observations. For a complete description of the observing and reduction techniques for the WET, see Nather et al. (1990).

We made all observations of V471 Tau through Johnson $U$ filters to minimize, as much as possible, the contamination of the light curve by light from the K2 V secondary. Even so, in the $U$ band, the white dwarf contributes only about $20 \%$ of the light of the system. Our integration time was $10 \mathrm{~s}$ on all the telescopes except for the CFHT, where it was $5 \mathrm{~s}$. We summed the data into $20 \mathrm{~s}$ bins before reduction and did not taper the ends of individual runs.

Figure 1 shows a light curve of V471 Tau obtained with the CFHT. Of all the telescopes in the network, only the CFHT yielded a signal-to-noise ratio large enough to see the variations of the white dwarf directly in the data. This remarkable light curve exhibits all of the known types of brightness changes exhibited by V471 Tau: the large-amplitude, longperiod variation is an orbital effect; the large spike on the right is a flare; the rapid drop at the end is white dwarf eclipse ingress; and the small, short-period undulations evident throughout most of the light curve are the white dwarf variations.

For our current analysis, we are interested only in the shortperiod variations, so we removed not only atmospheric extinction effects from our light curves, but also all the effects caused by the presence of the red star. Furthermore, while we were able to acquire a comparison star bright enough in the $U$ band to serve as a continuous monitor of photometric conditions, the comparison star brightness was too close to sky back-

TABLE 1

JOURNAL OF OBSERVATIONS

\begin{tabular}{|c|c|c|c|c|c|c|c|c|c|}
\hline Run Name & Telescope & $\begin{array}{c}\text { Date } \\
\text { (1988 UT) }\end{array}$ & $\begin{array}{l}\text { Start } \\
\text { (UT) }\end{array}$ & $\begin{array}{l}\text { Length } \\
\text { (s) }\end{array}$ & Run Name & Telescope & $\begin{array}{c}\text { Date } \\
\text { (1988 UT) }\end{array}$ & $\begin{array}{l}\text { Start } \\
\text { (UT) }\end{array}$ & $\begin{array}{l}\text { Length } \\
\text { (s) }\end{array}$ \\
\hline S4454 ......... & $\mathrm{SAAO}^{\mathrm{a}} 0.75 \mathrm{~m}$ & Nov 5 & $1: 26: 01$ & 2685 & GV-0033 .. & OHP $1.9 \mathrm{~m}$ & Nov 13 & $20: 55: 20$ & 24850 \\
\hline ESO $1 \ldots \ldots \ldots$ & Walraven $1 \mathrm{~m}$ & Nov 5 & $5: 47: 08$ & 9809 & CFC-0007 & McDonald $0.9 \mathrm{~m}$ & Nov 14 & $1: 35: 10$ & 3000 \\
\hline ESO $2 \ldots \ldots \ldots$ & Walraven $1 \mathrm{~m}$ & Nov 6 & $5: 33: 02$ & 10577 & CFC-0008 .... & McDonald $0.9 \mathrm{~m}$ & Nov 14 & $2: 39: 10$ & 2240 \\
\hline MAW-0018 .... & McDonald $2.1 \mathrm{~m}$ & Nov 6 & $7: 15: 40$ & 16770 & MAW-0034 .... & McDonald $2.1 \mathrm{~m}$ & Nov 14 & $3: 13: 10$ & 19770 \\
\hline ESO $3 \ldots \ldots \ldots$ & Walraven $1 \mathrm{~m}$ & Nov 7 & $6: 07: 05$ & 3457 & A95 ............ & Mauna Kea 0.6 m & Nov 14 & $10: 05: 30$ & 1670 \\
\hline MAW-0020 .... & McDonald $2.1 \mathrm{~m}$ & Nov 7 & $8: 41: 30$ & 11210 & S4460......... & SAAO $0.75 \mathrm{~m}$ & Nov 14 & $20: 13: 40$ & 3990 \\
\hline REN-0041 ..... & Siding Spring $1.0 \mathrm{~m}$ & Nov 7 & $16: 01: 50$ & 5190 & S4461 .......... & SAAO $0.75 \mathrm{~m}$ & Nov 14 & $21: 34: 50$ & 12410 \\
\hline ESO $4 \ldots \ldots \ldots$ & Walraven $1 \mathrm{~m}$ & Nov 8 & $6: 08: 35$ & 8140 & TMK-0028 ... & Kavalur $1 \mathrm{~m}$ & Nov 14 & $21: 51: 46$ & 7170 \\
\hline MAW-0022 .... & McDonald $2.1 \mathrm{~m}$ & Nov 8 & $8: 11: 40$ & 13360 & GV-0035 ...... & OHP $1.9 \mathrm{~m}$ & Nov 14 & $23: 25: 30$ & 15600 \\
\hline $\mathrm{A} 86 \ldots \ldots \ldots \ldots$ & Mauna Kea 0.6 m & Nov 8 & $10: 24: 00$ & 14890 & MAW-0035 .... & McDonald $2.1 \mathrm{~m}$ & Nov 15 & $4: 01: 10$ & 28730 \\
\hline REN-0043 ..... & Siding Spring $1.0 \mathrm{~m}$ & Nov 8 & $15: 47: 40$ & 2200 & A96 ............ & Mauna Kea $0.6 \mathrm{~m}$ & Nov 15 & $12: 04: 00$ & 680 \\
\hline ESO $5 \ldots \ldots \ldots$ & Walraven $1 \mathrm{~m}$ & Nov 9 & $3: 16: 43$ & 18286 & TMK-0029 ... & Kavalur $1 \mathrm{~m}$ & Nov 15 & $15: 00: 00$ & 9810 \\
\hline MAW-0025 .... & McDonald $2.1 \mathrm{~m}$ & Nov 9 & $8: 42: 00$ & 11710 & TMK-0032 ... & Kavalur $1 \mathrm{~m}$ & Nov 15 & $18: 33: 50$ & 775 \\
\hline A87 ............ & Mauna Kea $0.6 \mathrm{~m}$ & Nov 9 & $13: 02: 00$ & 7780 & TMK-0034 ... & Kavalur $1 \mathrm{~m}$ & Nov 15 & $21: 20: 50$ & 9500 \\
\hline TMK-0021 ... & Kavalur $1 \mathrm{~m}$ & Nov 9 & $20: 15: 30$ & 9240 & S4464 ......... & SAAO $0.75 \mathrm{~m}$ & Nov 15 & $22: 34: 00$ & 8950 \\
\hline MAW-0027 .... & McDonald $2.1 \mathrm{~m}$ & Nov 10 & $9: 04: 40$ & 10170 & CFC-0012 ..... & McDonald $0.9 \mathrm{~m}$ & Nov 16 & $1: 24: 10$ & 24270 \\
\hline ESO $6 \ldots \ldots \ldots$ & Walraven $1 \mathrm{~m}$ & Nov 10 & $1: 08: 34$ & 25786 & MAW-0037 .... & McDonald $2.1 \mathrm{~m}$ & Nov 16 & $7: 57: 20$ & 14590 \\
\hline A89 ........... & Mauna Kea 0.6 m & Nov 10 & $10: 50: 30$ & 5870 & A98 ............ & Mauna Kea $0.6 \mathrm{~m}$ & Nov 16 & $11: 54: 00$ & 11200 \\
\hline REN-0046 ..... & Siding Spring $1.0 \mathrm{~m}$ & Nov 10 & $15: 46: 00$ & 9490 & TMK-0035 ... & Kavalur $1 \mathrm{~m}$ & Nov 16 & $18: 33: 00$ & 19000 \\
\hline TMK-0024 .... & Kavalur $1 \mathrm{~m}$ & Nov 10 & $16: 41: 20$ & 17780 & CFC-0014 ..... & McDonald $0.9 \mathrm{~m}$ & Nov 17 & $1: 19: 00$ & 17270 \\
\hline ESO $7 \ldots \ldots \ldots$ & Walraven $1 \mathrm{~m}$ & Nov 11 & $1: 05: 23$ & 26046 & TMK-0036 ... & Kavalur $1 \mathrm{~m}$ & Nov 17 & $15: 00: 00$ & 29260 \\
\hline REN-0047 .... & Siding Spring $1.0 \mathrm{~m}$ & Nov 11 & $11: 40: 10$ & 3740 & S4467 ......... & SAAO $0.75 \mathrm{~m}$ & Nov 17 & $21: 41: 00$ & 6460 \\
\hline REN-0048 ..... & Siding Spring $1.0 \mathrm{~m}$ & Nov 11 & $12: 48: 40$ & 19630 & MAW-0041 .... & McDonald $2.1 \mathrm{~m}$ & Nov 18 & $8: 12: 30$ & 13770 \\
\hline TMK-0025 ... & Kavalur $1 \mathrm{~m}$ & Nov 11 & $17: 31: 29$ & 1400 & TMK-0037 .... & Kavalur $1 \mathrm{~m}$ & Nov 18 & $15: 00: 00$ & 17420 \\
\hline S4458 ......... & SAAO $0.75 \mathrm{~m}$ & Nov 11 & $21: 06: 40$ & 17890 & TMK-0038 $\ldots$. & Kavalur $1 \mathrm{~m}$ & Nov 18 & $19: 51: 00$ & 14160 \\
\hline GV-0031 ...... & $\mathrm{OHP}^{\mathrm{b}} 1.9 \mathrm{~m}$ & Nov 11 & $22: 51: 00$ & 11490 & S4469 .......... & SAAO $0.75 \mathrm{~m}$ & Nov 18 & 20:04:00 & 17100 \\
\hline ESO $8 \ldots \ldots \ldots$ & Walraven $1 \mathrm{~m}$ & Nov 12 & $1: 35: 00$ & 24306 & CFC-0015 ..... & McDonald $0.9 \mathrm{~m}$ & Nov 19 & $1: 13: 40$ & 27210 \\
\hline S4459 ........... & SAAO $0.75 \mathrm{~m}$ & Nov 12 & $21: 10: 10$ & 15390 & TMK-0039 .... & Kavalur $1 \mathrm{~m}$ & Nov 19 & $15: 00: 00$ & 31900 \\
\hline MAW-0030 .... & McDonald $2.1 \mathrm{~m}$ & Nov 12 & $3: 49: 30$ & 29130 & MLF-0002 ..... & McDonald $0.8 \mathrm{~m}$ & Nov 20 & $7: 05: 40$ & 9310 \\
\hline A92 ........... & Mauna Kea 0.6 m & Nov 12 & $10: 31: 00$ & 16260 & TMK-0041 ... & Kavalur $1 \mathrm{~m}$ & Nov 20 & $14: 00: 00$ & 34230 \\
\hline REN-0050 ..... & Siding Spring $1.0 \mathrm{~m}$ & Nov 12 & $14: 15: 20$ & 5850 & S4471 .......... & SAAO $0.75 \mathrm{~m}$ & Nov 20 & $23: 00: 00$ & 6460 \\
\hline REN-0051 .... & Siding Spring $1.0 \mathrm{~m}$ & Nov 12 & $16: 12: 19$ & 6360 & S4473 ........... & SAAO $0.75 \mathrm{~m}$ & Nov 21 & $21: 18: 00$ & 2110 \\
\hline REN-0054 ..... & Siding Spring $1.0 \mathrm{~m}$ & Nov 13 & $12: 00: 00$ & 22470 & JCC-0064 ...... & $\mathrm{CFHT}^{\mathrm{c}} 3.6 \mathrm{~m}$ & Dec 1 & $8: 58: 30$ & 5260 \\
\hline CFC-0004 ..... & McDonald $0.9 \mathrm{~m}$ & Nov 13 & $1: 33: 30$ & 4950 & JCC-0067 ...... & CFHT $3.6 \mathrm{~m}$ & Dec 2 & $7: 55: 00$ & 23860 \\
\hline MAW-0032 .... & McDonald $2.1 \mathrm{~m}$ & Nov 13 & $3: 17: 50$ & 31330 & JCC-0068 ..... & CFHT $3.6 \mathrm{~m}$ & Dec 3 & $5: 45: 30$ & 26475 \\
\hline A94 ............ & Mauna Kea 0.6 m & Nov 13 & $10: 30: 10$ & 4210 & & & & & \\
\hline
\end{tabular}

a South African Astronomical Observatory.

b Observatoire Haute Provence.

c Canada-France-Hawaii Telescope. 


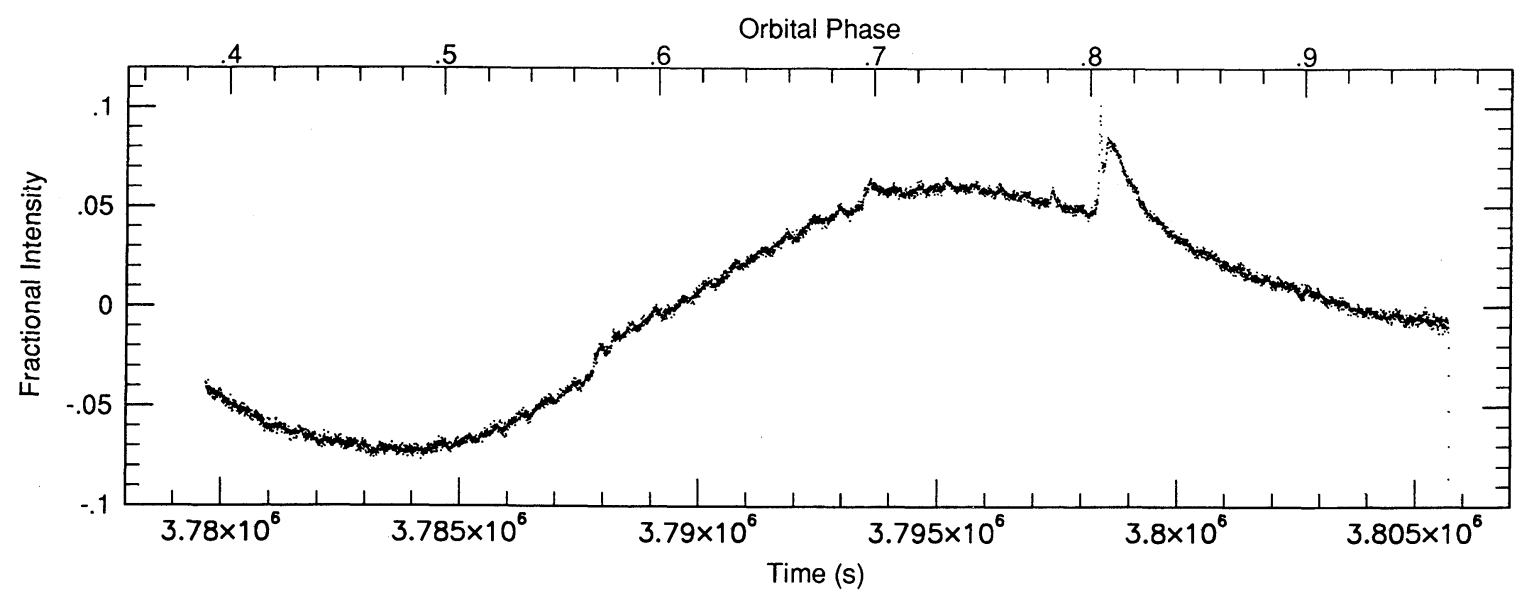

FIG. 1.-U-band light curve of V471 Tau acquired with the $3.6 \mathrm{~m}$ CFHT

ground to provide an accurate measure of extinction (except in the CFHT data). Since we had no good model for the combined extinction and orbital modulations, we simply removed the long-term variations in the star by fitting low-order polynomials to the data, dividing by those polynomials, and subtracting one. This left the data with a mean of zero and amplitudes expressed as a fraction of the mean total brightness of the binary. Whenever flares or eclipses interrupted the data, we discarded those portions of the light curve and reduced each remaining section as a separate run. Our complete, reduced data set contains $131 \mathrm{hr}$ of WET data and $12 \mathrm{hr}$ of follow-up observations.

\section{ANALYSIS I: THE FOURIER TRANSFORM}

Previous observations of V471 Tau by Robinson et al. (1988) and Winget \& Claver (1989) have hinted, but have not conclusively demonstrated, that periods other than $555 \mathrm{~s}$ and its $277 \mathrm{~s}$ harmonic are present in the light curve of the binary. Multiperiodicity is a signature of $g$-mode pulsations but not of rotational effects, which typically generate signals at only the rotational period and its harmonics. Consequently, establishing whether additional periods exist is vital to learning whether $g$-modes are present.

We have calculated the Fourier transform (FT) of the WET data to search for the presence of additional periods. Figure 2 shows the amplitude spectrum of our data for frequencies between 0 and $12,500 \mu \mathrm{Hz}$. In this FT we find significant peaks at only three frequencies. Two of these correspond to the 555 and $277 \mathrm{~s}$ periodicities previously known to be generated by the white dwarf in the binary; the third is a newly detected signal with a period of $561.59 \mathrm{~s}$. We do not see any evidence for a signal at $410 \mathrm{~s}$ as reported by Winget $\&$ Claver (1989).

Figures $3 a$ and $3 b$ enlargements of the regions of the amplitude spectrum in which the significant peaks occur. Arrows mark the peaks we consider significant. For each of the three frequencies we detected, we used a nonlinear least-squares estimation to find the period, amplitude, and time of maximum of the sine wave which best fit the WET data. Table 2 lists these quantities along with the formal errors from the least-squares fit. We regard these errors only as estimates since the noise in our light curves is not Gaussian.

To be certain that no additional peaks are hidden in the regions around 555 and $277 \mathrm{~s}$, we subtracted two sine waves from the reduced data and recalculated the FT. The sine waves we subtracted had the same period, amplitude, and phase as those listed in Table 2 for the 555 and 277 s peaks. Figures $3 c$ and $3 d$ show the result. No significant peaks remain in the region around $177 \mathrm{~s}$ (Fig. $3 d$ ) and only the $562 \mathrm{~s}$ peak and its sidelobes remain in the region near $555 \mathrm{~s}$ (Fig. 3c). For reference, the inset in Figure $3 d$ shows the pattern generated when we calculate the FT of a single artificial sine wave sampled exactly as the data were sampled (we refer to this as the "spectral window"). The horizontal scale of the spectral window is the same as that for the larger panels.

At first, our detection of a signal which is not a harmonic of $555 \mathrm{~s}$ seems to imply that pulsations are present. Further reflection shows this conclusion to be unwarranted; even if we invoke a rotational model to explain the 555 and $277 \mathrm{~s}$ variations of the white dwarf, a signal can occur at $562 \mathrm{~s}$ as a consequence of the presence of the white dwarf's orbital companion. We explain why in the discussion which follows.

\section{DISCUSSION OF THE FOURIER TRANSFORM}

For the purpose of this discussion, we will assume that the $555 \mathrm{~s}$ signal and its harmonic are created when bright or dark regions on the surface of the white dwarf rotate in and out of our view. This does not mean we have settled on this as the preferred model. We simply wish to show how the $562 \mathrm{~s}$ period arises as a natural consequence of this model.

Young \& Skumanich (1983) have found variability in the $\mathrm{H} \alpha$ line of the K star in V471 Tau. Specifically, the line changes from absorption near primary eclipse to emission when the side of the $\mathrm{K}$ star illuminated by the white dwarf faces us. Young, Skumanich, \& Paylor (1988) interpret this as reprocessing of radiation from the hot white dwarf via fluorescence.

TABLE 2

Peaks in the Amplitude Spectrum of the WeT Data

\begin{tabular}{cccc}
\hline \hline & & $\begin{array}{c}\text { Fractional } \\
\text { Amplitude }\end{array}$ & \\
$\begin{array}{c}\text { Frequency } \\
(\mu \mathrm{Hz})\end{array}$ & $\begin{array}{c}\text { Period } \\
\text { in Units of } \\
10^{-4}\end{array}$ & $\begin{array}{c}\text { Time of Maximum } \\
(\mathrm{BJDD}-2,440,000)\end{array}$ \\
\hline $1780.66 \ldots \ldots$ & $561.59 \pm 0.02$ & $4.6 \pm 0.7$ & $7471.9658 \pm 0.0003$ \\
$1803.00 \ldots \ldots$ & $554.63 \pm 0.01$ & $9.5 \pm 0.7$ & $7471.9641 \pm 0.0002$ \\
$3605.96 \ldots \ldots$ & $277.319 \pm 0.003$ & $7.0 \pm 0.7$ & $7471.9644 \pm 0.0001$ \\
\hline
\end{tabular}




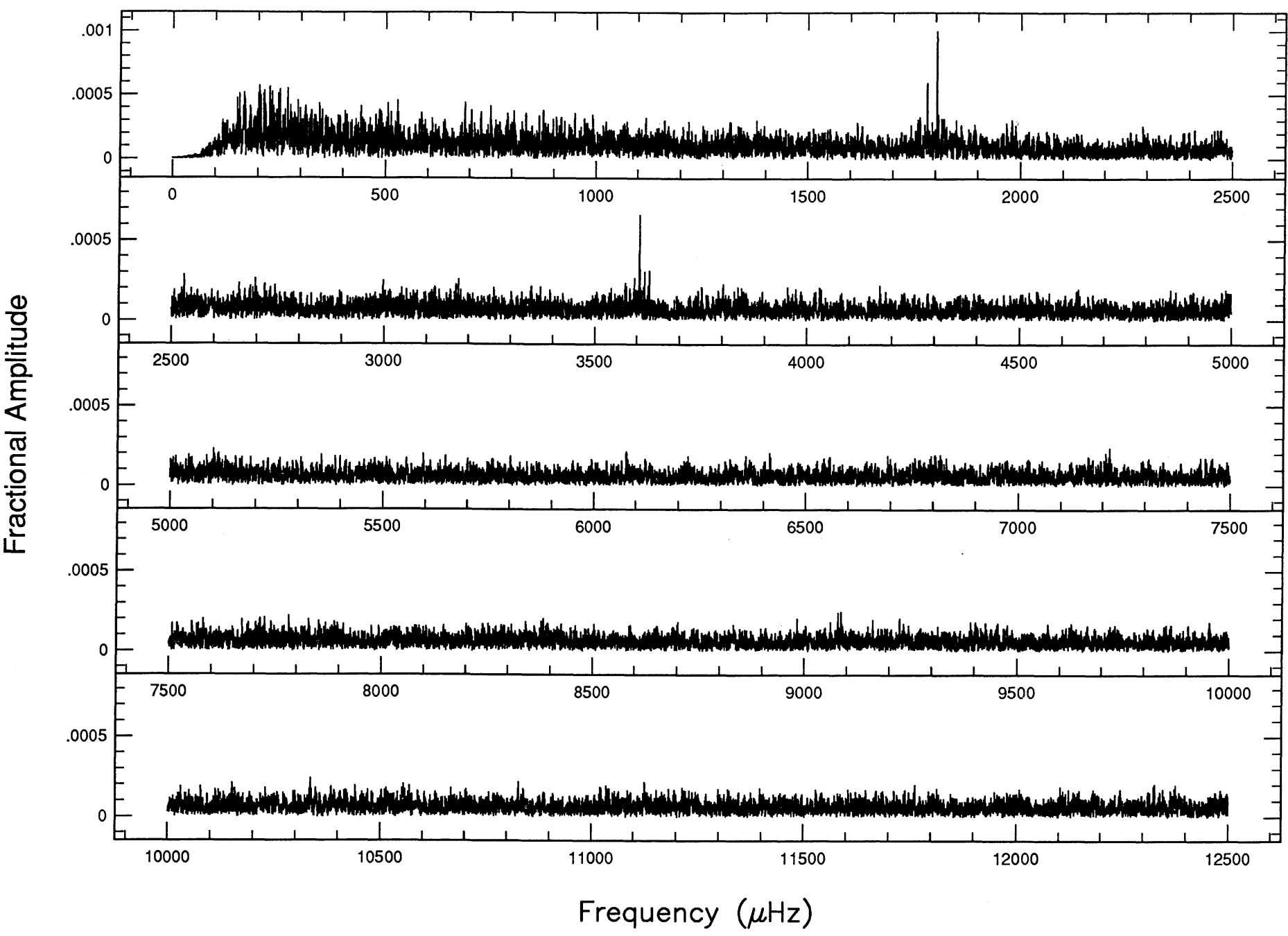

FIG. 2.-Amplitude spectrum of the V471 Tau WET data

According to Young et al., on the illuminated side of the $\mathrm{K}$ star, high-energy photons from the white dwarf ionize hydrogen in the chromosphere, which then reemits the excess energy as line radiation during recombination. A significant fraction of this radiation should be Balmer continuum radiation, whose wavelength lies within the Johnson $U$ bandpass. Hence the reprocessed radiation is, in principle, observable via $U$-band photometry.

From the X-ray data of Jensen et al. (1986), we know that at least some of the white dwarf radiation capable of ionizing hydrogen is pulsed. Whether or not the reprocessed radiation retains this pulsed character depends upon the reprocessing timescale. In the Appendix, we estimate this time scale to be about $1 \mathrm{~s}$. This is short compared to $555 \mathrm{~s}$, so the reprocessed radiation should be pulsed. This does not guarantee that the reprocessed pulses have observable amplitude.

If the pulsed reprocessed radiation is observable, we do not expect it to have the same period as the white dwarf pulses (Patterson \& Price 1981). Figure 4 is a schematic of V471 Tau which shows why. The dot in the center of each panel represents the white dwarf and the straight, solid arrow represents a single feature on its surface. We assume for now that the rotation axis of the white dwarf is parallel to the axis of the binary orbit, and that the rotation is prograde with a period of $555 \mathrm{~s}$, much less than the orbital period of 0.521 days.

Observed at orbital phase 0.0 , the feature represented by the arrow points at the $\mathrm{K}$ star (generating a reprocessed pulse) and toward the observer at the same time. If we ignore the small light travel time across the orbit (approximately $7 \mathrm{~s}$ ) and the reprocessing delay, the direct and reprocessed pulses are in phase. Of course the observer can see neither because of the eclipse.

At orbital phase 0.25 , the arrow points first at the observer and then, after the white dwarf has turned through one-fourth of its rotation, at the $\mathrm{K}$ star. Thus the reprocessed pulse lags behind the direct pulse by one-fourth of the white dwarf's rotation period.

At phase 0.5 , the delay between direct and reprocessed pulse has increased to half the rotation period of the white dwarf, and at 0.75 the delay is three-fourths of the white dwarf's rotation period. By the time one full orbit is complete, the delay has become one full white dwarf rotation period and the two pulses are again in phase. This demonstrates that, for the assumed prograde rotation, the period of the reprocessed pulse should 


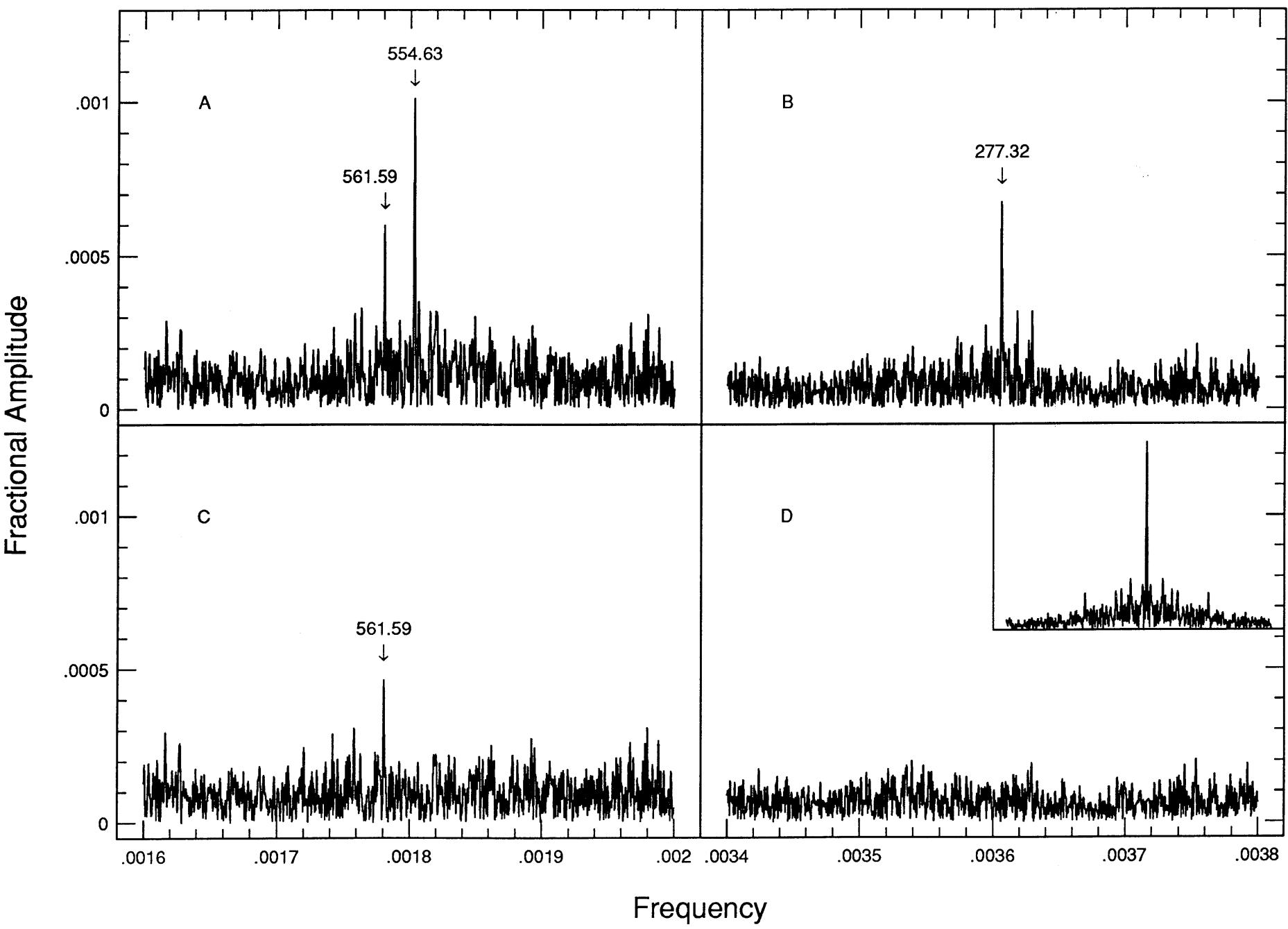

Fig. 3.-Enlarged amplitude spectra of V471 Tau WET data. Fig. $3 c$ and Fig. $3 d$ show the residuals after the periodicities at $555 \mathrm{~s}$ and $277 \mathrm{~s} \mathrm{have} \mathrm{been} \mathrm{removed.}$ The inset shows the spectral window.

be longer than that of the direct pulse. The amount by which the period differs is related to the orbital period by the expression

$$
\frac{1}{P_{r}}=\frac{1}{P_{d}}-\frac{1}{P_{\text {orb }}},
$$

where $P_{r}$ is the period of the reprocessed pulse, $P_{d}$ is the period of the direct pulse, and $P_{\text {orb }}$ is the orbital period. If we substitute the known orbital period of V471 Tau and the exact period of the $555 \mathrm{~s}$ variations into this equation, we find that the period of any reprocessed signal should be $561.55 \mathrm{~s}$ - very close to the new period of $561.59 \mathrm{~s}$ we have discovered in V471 Tau.

If the signal we have discovered is reprocessed radiation from the chromosphere of the $\mathrm{K}$ star, then we do not expect it to have constant amplitude. Its amplitude should be much larger when the side of the $\mathbf{K}$ star which is illuminated by the white dwarf faces us than when the illuminated side faces away from us. We have analyzed our data to see how the amplitude of the $562 \mathrm{~s}$ variation behaves with respect to orbital phase.
The low signal-to-noise ratio forces us to rely on signal averaging techniques for this analysis.

First we made a linear least-squares fit to the times of mideclipse measured from our data to acquire an updated orbital ephemeris for the binary. The resulting oibital period and time of mid-eclipse are $P_{\text {orb }}=0.5211831 \pm 0.0000002$ days and $T_{\text {mid-eclipse }}=2,447,471.962896 \pm 0.000005$ Barycentric Julian Dynamical Date (BJDD; see Kepler et al. 1982).

We then divided our data into two sets according to this ephemeris: one including observations from the half of the orbit centered on primary eclipse (orbital phase $0.75-0.25$ ); and the other including the opposite half of the orbit (orbital phase $0.25-0.75$ ). To make detection of the $562 \mathrm{~s}$ signal more straightforward, we subtracted a sine wave with the same period and phase shown in Table 2 for the $555 \mathrm{~s}$ variations. Since a sidelobe of the $562 \mathrm{~s}$ signal lies at exactly the same frequency as the $555 \mathrm{~s}$ peak, we adjusted the amplitude of the sine wave we subtracted until the peak which remained at $555 \mathrm{~s}$ had the same amplitude as the corresponding sidelobe on the low-frequency side of the 562 s peak.

Figure 5 shows the result. Figure $5 a$ contains the amplitude spectrum of the data centered on eclipse, when the side of the 


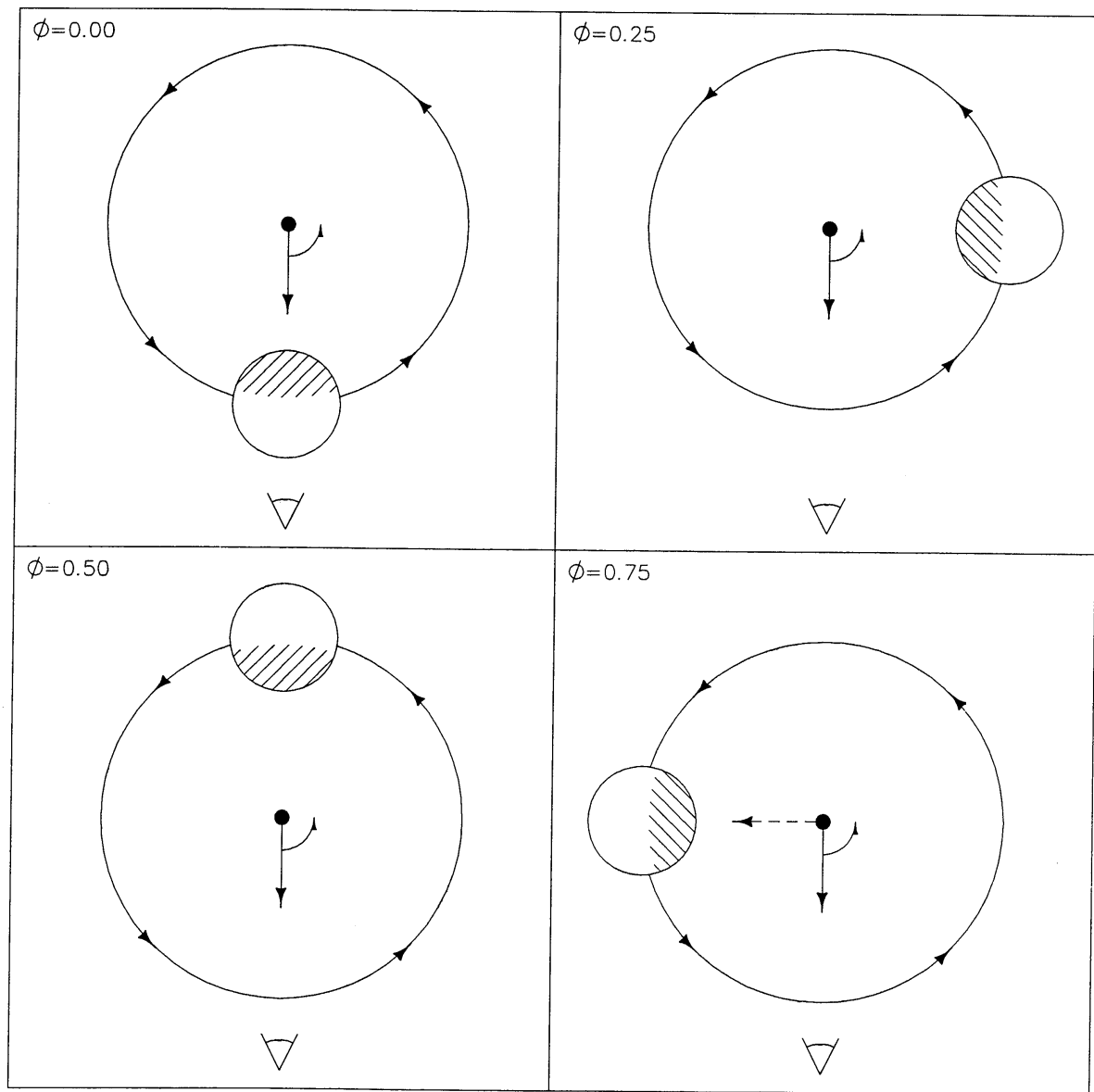

FIG. 4.-Schematic of the rotational model for V471 Tauri at four different orbital phases

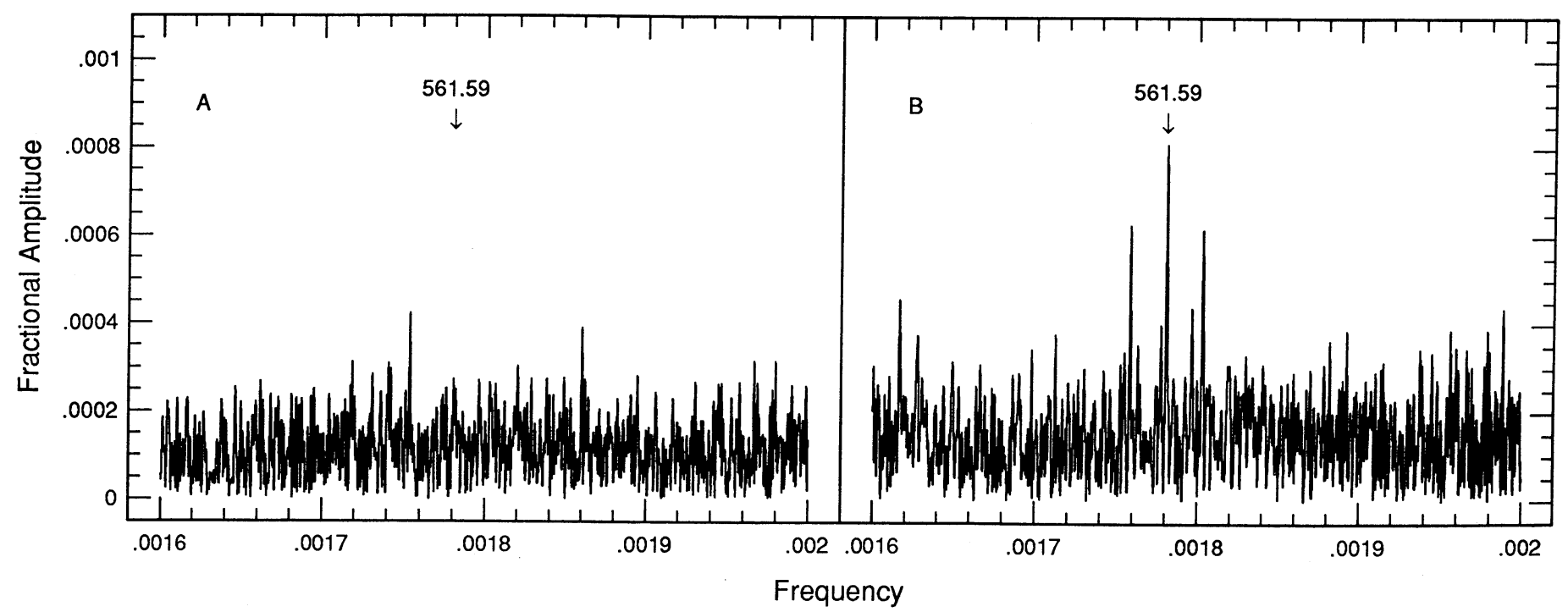

FIG. 5.-Amplitude spectra of WET data for two different orbital phases, with the $555 \mathrm{~s}$ signal subtracted 
secondary illuminated by the white dwarf faces away from us. There is no evidence for a significant peak at $562 \mathrm{~s}$. Conversely, in the amplitude spectrum of the data set which includes the half of the orbit opposite primary eclipse (Fig. $5 b$ ), the $562 \mathrm{~s}$ period is quite large. Its sidelobes are caused by a combination of the aliases introduced by using data from only half of the orbit, and the sidelobes generated by amplitude modulation of the signal. From Figure 5, we conclude that the amplitude of the $562 \mathrm{~s}$ signal behaves as we expect for reprocessed radiation; it is large when the side of the $\mathrm{K}$ star illuminated by the white dwarf faces us and small otherwise.

We have now shown that the $562 \mathrm{~s}$ signal has both the correct period and the correct amplitude modulation to be reprocessed radiation. Furthermore, we know from the spectroscopy of Young et al. (1988) that reprocessing occurs in V471 Tau. Together, these facts lead us to conclude that the $562 \mathrm{~s}$ period in the light curve of V471 Tau is due to reprocessing.

Identification of the $562 \mathrm{~s}$ period as reprocessing of the white dwarf variations by the $\mathrm{K}$ star does not tell us the cause of the 555 and $277 \mathrm{~s}$ signals. Throughout this discussion we have assumed that they are due to rotation of the white dwarf, but this is not a necessary assumption. $g$-mode pulsations could conceivably generate reprocessed radiation with the same period and amplitude behavior we have observed. To choose the correct model for the white dwarf variations, we must extend our analysis.

\section{ANALYSIS II: PULSE SHAPES}

Since the FT did not reveal the cause of the white dwarf variations, we continued our analysis by folding the data at the $555 \mathrm{~s}$ period to create an average pulse shape. A pulse shape constructed in this way does not contain any information not included in the FT, but its noise properties are much different. Coherent harmonics of the $555 \mathrm{~s}$ peak which are hidden by noise in the FT can appear in the pulse shape, since averaging many cycles tends to reduce the noise with respect to the signal.

It is useful to fold our data at $555 \mathrm{~s}$ only if the signal with that period is not changing its phase on time scales shorter than the length of our data set. To see how the phase behaved during our observations, we constructed an $O-C$ diagram for the 555 and $277 \mathrm{~s}$ variations. Figure 6 is a plot of the difference between the times of maxima of the 555 and $277 \mathrm{~s}$ variations observed in individual runs and the times of maxima calculated using linear ephemerides based on values in Table 2. The linear shape of the $O-C$ plot demonstrates that the period and phase of both the 555 and $277 \mathrm{~s}$ variations remained essentially constant for the duration of our observations (the motion of the white dwarf about the center of mass of the binary introduces a periodic variation in the $O-C$, but its amplitude is only about $4 \mathrm{~s}$ - too small to be seen in our $O-C$ diagram).

Once we knew that the $555 \mathrm{~s}$ variations did not change in period or phase during the time we observed, we constructed an average pulse shape by folding the data from the two largest telescopes ( 2.1 and $3.6 \mathrm{~m})$ at $554.634 \mathrm{~s}$, our best estimate for the basic period. The top panel of Figure 7 shows the result-a double-pulsed curve with maxima of unequal height but minima of roughly equal depth.

To estimate the noise in this pulse shape, we folded the same data set used to create it at a period incommensurate with 555 and $562 \mathrm{~s}$ and measured the variance of the result. The square

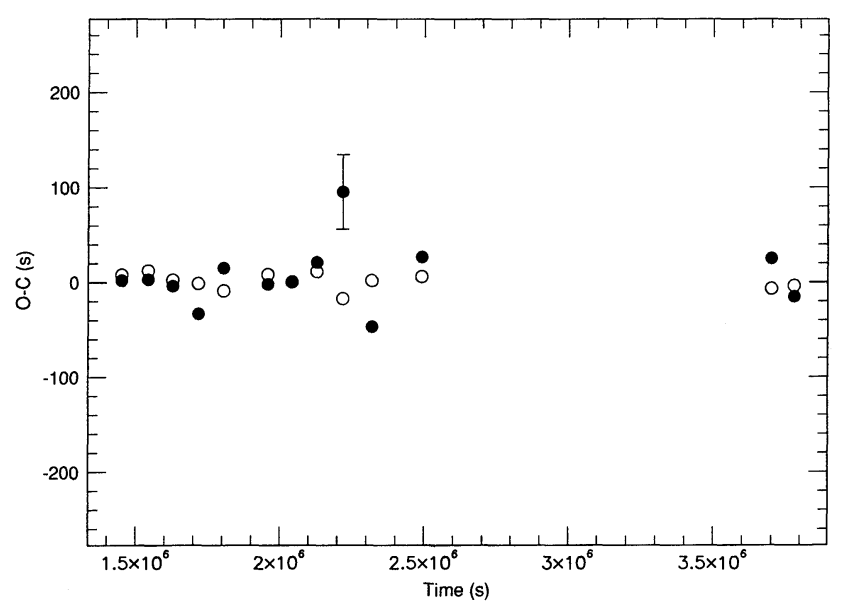

FIG. 6.- $O-C$ diagram for WET and follow-up data. Open circles represent $O-C$ values for the $277 \mathrm{~s}$ signal; closed circles represent the signal at $555 \mathrm{~s}$. The error bar is for the largest error. The smallest errors are less than the size of the circles.

root of the value measured, $9.3 \times 10^{-5}$, is a good estimate of the noise present in the average pulse shape for the $555 \mathrm{~s}$ signal, in units of fractional intensity. We also folded the 2.1 and $3.6 \mathrm{~m}$ telescope data sets independently, to see if the pulse shape was stable. The resulting pulse shapes did not differ significantly from the one pictured in the top panel of Figure 7.

We constructed a pulse shape for the reprocessed pulse by folding the data at $561.59 \mathrm{~s}$. For this test we did not use the entire large telescope data set; instead, we divided the data by orbital phase and included only data from the half of the orbit where the amplitude of the reprocessed signal was large (orbital phase $0.25-0.75$ ). The middle panel of Figure 7 shows the resulting pulse shape. The estimated noise present in this pulse shape is $1.3 \times 10^{-4}$.

Finally, we have extracted X-ray data from the EXOSAT archives and calculated the $555 \mathrm{~s} X$-ray pulse shape using the same procedure as for the $555 \mathrm{~s}$ optical pulse shape, The data we used are EXOSAT observations acquired on 1985 August 22 with the LE1 (low-energy) telescope through the thin Lexan filter. The bottom panel of Figure 7 contains the X-ray pulse shape.

\section{DISCUSSION OF PULSE SHAPES}

\subsection{The 555 Second Pulse Shape}

The pulse shape we have measured for the $555 \mathrm{~s}$ variations does not resemble the pulse shape of any known pulsating white dwarf. Large-amplitude $g$-mode pulsations in white dwarfs have pulse profiles with sharp maxima and broader minima (see, for example, Winget, Nather, \& Hill 1987), while small-amplitude pulsations have sinelike profiles. Furthermore, $g$-mode pulsators usually exhibit one or more sets of closely spaced modes. Beating between these modes modulates the amplitude of the pulses on time scales much shorter than the duration of our observations. Nonetheless, it is possible to construct a set of modes, however unlikely, which would create the pulse shape we have measured. Thus we cannot rule out pulsations on the basis of the pulse shape alone.

In contrast to the pulsation model, the magnetic accretor model explains the measured pulse shape quite naturally. We expect accretion onto the magnetic poles of a white dwarf to 


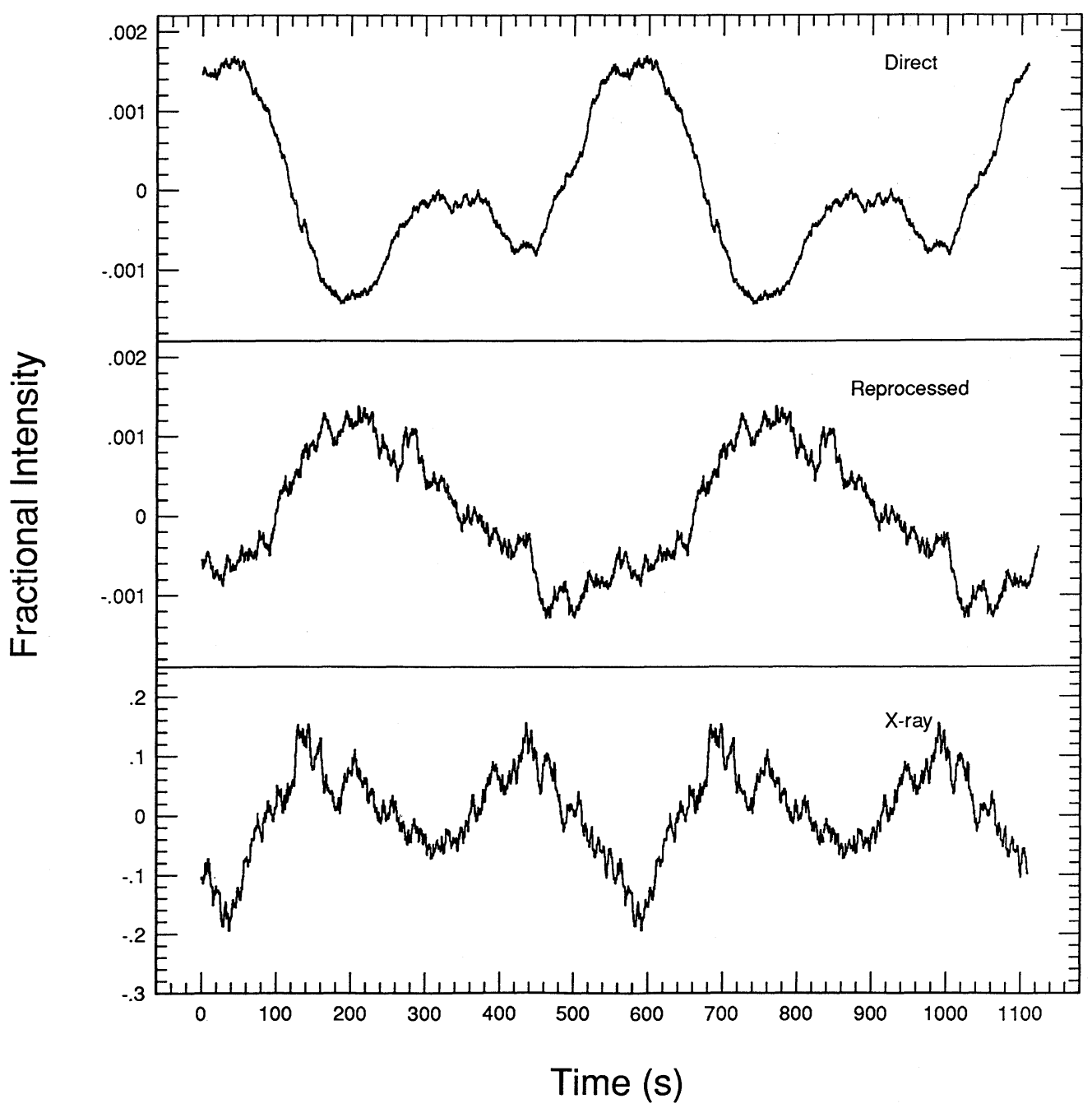

Fig. 7.-Average pulse shape of the direct $U$-band variations, the reprocessed variations, and the $\mathrm{X}$-ray variations. We have plotted two complete cycles for clarity.

create regions which are bright at optical wavelengths. If the magnetic poles are misaligned with the rotation axis, then the bright regions can rotate in and out of view. Two bright regions on opposite poles of the white dwarf will create the pulse shape we observe if one of the regions is brighter than the other or if the rotation axis is not perpendicular to our line of sight. We consider this model to be much more likely than pulsations but reserve our final conclusion until after we have considered the essential information provided by the reprocessed pulses.

\subsection{The 562 Second Pulse Shape}

The best test we could perform to determine the nature of the white dwarf variations would be to compare the phase of the optical pulses directly to the phase of the X-ray pulses. If the variations are due to $g$-mode pulsations then we would find that the phases are identical, because $g$-mode pulsations modulate the brightness of a star by creating temperature changes in the photosphere, and therefore must have the same phase at all wavelengths (Robinson, Kepler, \& Nather 1982). If the variations are due to accretion onto a magnetic rotator, then the best model predicts that the X-ray and optical pulses should be out of phase. If this model is correct, we would find that the $\mathrm{X}$-ray minima coincide with the optical maxima, because the accretion poles are darkened in $\mathrm{X}$-ray wavelengths and brightened in optical.

Unfortunately we cannot currently make a direct comparison, because neither the X-ray nor the optical pulse period is precise enough to bridge the gap which separates the X-ray data from the optical data. However, our discovery of the reprocessed radiation permits us to make an indirect comparison. Because the reprocessed radiation we measure is generated when radiation of shorter wavelength from the white dwarf illuminates the $\mathrm{K}$ star, the reprocessed pulses carry crucial information about the shorter wavelength light which produced them. We can use this information to measure the phase of the X-ray variations and then compare that phase to the optical phase. Before we do this, we will first address a peculiar feature of the $562 \mathrm{~s}$ pulse shape.

Because the reprocessed signal is caused by shortwavelength radiation from the white dwarf which includes the soft X-rays, we expect the reprocessed pulses to have a shape similar to the X-ray pulses. However, the reprocessed pulse 
shape pictured in the middle panel of Figure 7 does not have the double-pulsed character displayed by the X-ray pulse shape. This explains why we see a peak due to reprocessed radiation at $562 \mathrm{~s}$ in the FT but not at $281 \mathrm{~s}$ as we might expect. We offer three possible reasons for the observed differences between the reprocessed and X-ray pulse shapes.

First, the X-ray pulse shape may have changed since 1985. It may now be shaped like the reprocessed signal it generates. Considering the double-pulsed nature of the $555 \mathrm{~s}$ optical pulses, we do not consider this to be very likely.

Second, if the rotation axis of the white dwarf is not parallel to the axis of the binary orbit, the $\mathrm{K}$ star will have a different view of the white dwarf at different orbital phases. If the reprocessing amplitude we see is large during orbital phases when the $\mathrm{K}$ star only sees one magnetic pole of the white dwarf, then the reprocessed radiation will not be double-pulsed. Considering that V471 Tau is an eclipsing binary, this geometry is almost impossible to contrive. If the $\mathrm{K}$ star only sees one magnetic pole at orbital phase opposite primary eclipse (when we see the reprocessing at it largest amplitude), then we are almost guaranteed to see only one magnetic pole as well. The direct optical and X-ray pulse shapes demonstrate that we see two. Thus we do not consider this explanation to be very likely either.

Finally, if the reprocessing is caused primarily by the ionization and recombination of hydrogen in the chromosphere of the $\mathrm{K}$ star, as we have supposed, then the soft X-rays which generate the X-ray pulse shape in Figure 7 are only a fraction of the radiation causing the reprocessing. Integrated over all the wavelengths which are capable of ionizing hydrogen, the pulse shape may not be so strongly double-pulsed as the X-ray pulse shape. We consider this the best explanation for the difference between the X-ray and reprocessed pulse shapes.

Whatever the reason for the pulse shape of the reprocessed signal, we can still use it to learn about the phase of the shorter wavelength radiation which created it. In our discussion of Figure 4 we mentioned that at orbital phase 0 (primary eclipse) our view of the white dwarf is the same as the K star's view. Thus measuring the phase of the reprocessed radiation near mid-eclipse will tell us the phase of the variations which created it. While we cannot observe the white dwarf during that phase because of eclipse, we can interpolate the phases we have measured for the 555 and $562 \mathrm{~s}$ variations to a time of mid-eclipse. When we perform this exercise, we find that the 555 and $562 \mathrm{~s}$ variations do not have the same phase at mideclipse. Instead, the time of maximum of the $555 \mathrm{~s}$ pulses is ahead of that for the $562 \mathrm{~s}$ pulses by $0.22 \pm 0.046$ cycles (roughly $\pi / 2 \mathrm{rad}$ ), implying that the phases of the 555 and 562 second pulses will coincide at orbital phase 0.78 .

This demonstrates that the shorter wavelength white dwarf variations which cause the reprocessing are not in phase with the $U$-band variations. The lower right-hand panel of Figure 4 illustrates this situation. As before, let the solid arrow represent a region on the surface of the white dwarf which is bright in the $U$ band. The dashed arrow represents a region which is bright at shorter wavelengths. When it faces the secondary, it generates a reprocessed pulse, while the region which is bright in the $U$-band does not. An observer who views the system at orbital phase 0.75 sees the direct pulse and the reprocessed pulse (from the $\mathrm{K}$ star) at the same time. Consequently, at orbital phase 0.0 , there is a $\pi / 2$ difference in the phase of the direct and reprocessed pulses, just as we observe.

Another way to see the phase difference between the direct and reprocessed radiation is to compare the pulse shapes in the top and middle panels of Figure 7. They were folded using the same epoch for the first bin-an epoch which also corresponds to a time of mid-eclipse. Thus the relative phase of the pulse shapes in the plot is the same as their relative phase at orbital phase 0 .

Furthermore, we cross-correlated the X-ray pulse shape with the reprocessed pulse shape and plotted the X-ray pulse shape using the phase which corresponds to maximum correlation with the reprocessed pulse shape. Therefore, to the degree that the phase of the reprocessed pulses represents the phase of the $\mathrm{X}$-ray pulses, the relative phases of the $U$-band and X-ray pulses from the white dwarf are exactly as they appear in Figure 7.

Clearly, as measured using the reprocessed radiation, the phase of the short-wavelength variations of the white dwarf in V471 Tau is not the same as the phase of the Jonhson $U$-band variations. As we discussed before, this proves conclusively that the variations cannot be due to $g$-mode pulsations, which must have the same phase at all wavelengths. Furthermore, the phase difference between the $U$ and X-ray variations is such that the maximum in $U$ coincides with the minimum in X-ray. This suggests that the variations are caused by regions on the surface of the white dwarf which are bright in $U$ wavelengths and dark in X-ray.

We have already mentioned that the Johnson $U$ pulse shape has unequal maxima and roughly equal minima. We point out that the X-ray pulse shape is the opposite; it has equal maxima and unequal minima. In Figure 8 we show the $U$ pulse shape along with the $\mathrm{X}$-ray pulse shape, which we have plotted upside down. The relative phase is the same as that in Figure 7. The remarkable similarity between the two shapes supports our claim that the regions causing the variations are bright in optical wavelengths and dark in X-ray wavelengths.

\section{CONCLUSIONS}

Among the models which have been proposed to explain the variations of the white dwarf in V471 Tau, only one is consistent with our observations. Based primarily on observations of the newly discovered reprocessed signal, we have concluded that the X-ray and optical modulations are not in phase. This eliminates from consideration any models which invoke $g$-mode pulsations or magnetic poles which are bright at both optical and X-ray wavelengths.

The model which remains is consistent with two important properties of V471 Tau: the secondary does not overflow its Roche lobe, and there is no disk around the white dwarf. We can explain our observations if the white dwarf accretes a relatively small amount of material from the wind of the K secondary. The magnetic field of the white dwarf channels the metal-rich material to the magnetic poles, where it settles, blocking flux at X-ray wavelengths and increasing flux in the $U$-band. These poles are misaligned with the rotation axis so, as the star rotates, first one and then the other pole moves into view, creating a double-pulsed light curve.

If this model is correct, then the rotation rate of the white dwarf is $554.63 \mathrm{~s}$. Furthermore, the direction of the rotation is prograde, since the reprocessed signal has lower frequency than the direct signal. In spite of this relatively high rotation rate, the propeller mechanism (see Illarionov \& Sunyaev 1975) apparently does not inhibit accretion along magnetic field lines. 


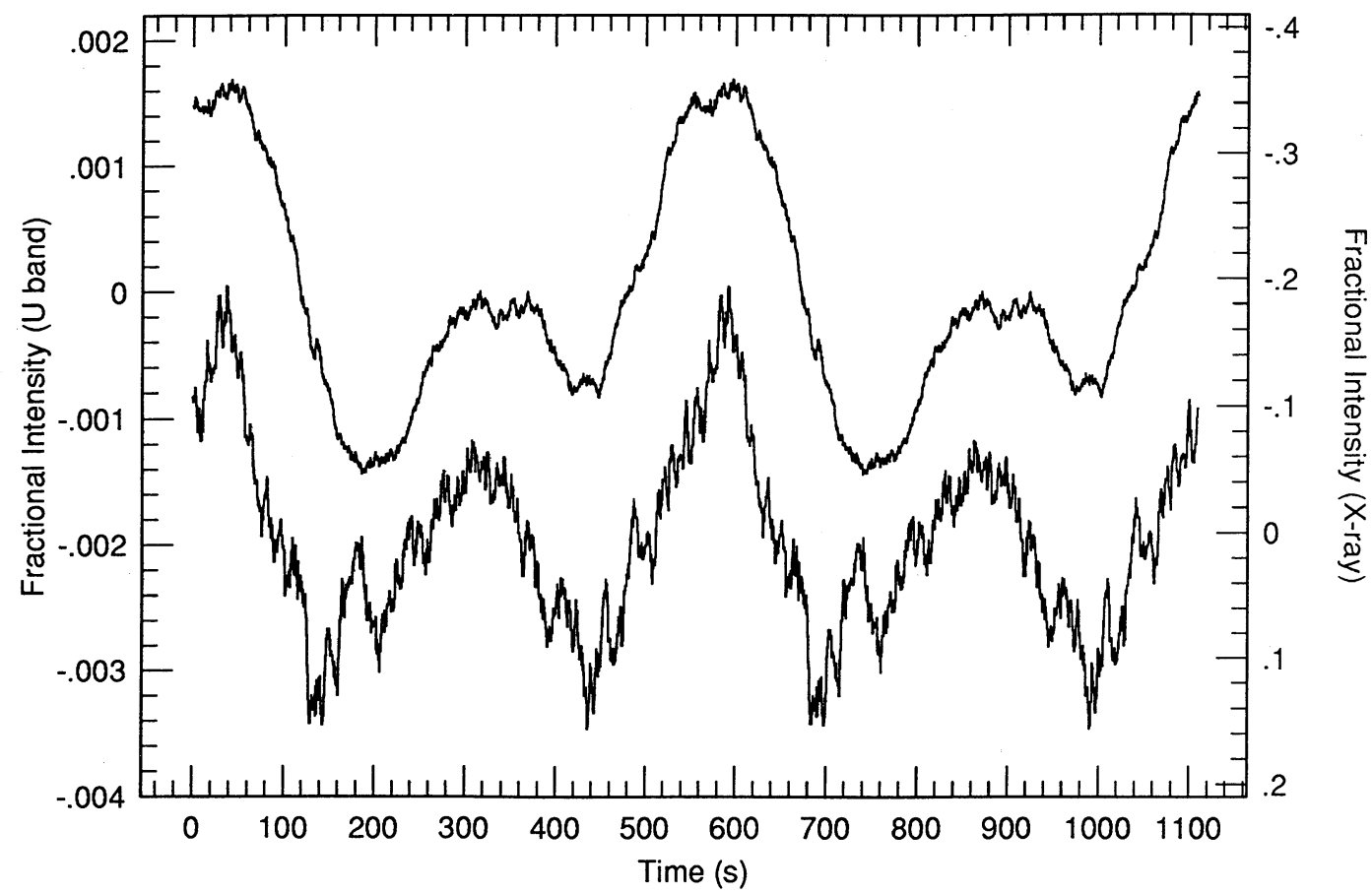

Fig. 8.-Direct comparison of the $\mathrm{X}$-ray and $U$-band pulse shapes. Note that the $\mathrm{X}$-ray pulse shape is plotted upside down.

As a magnetic accretor without a disk and without a synchronously rotating white dwarf, V471 Tau is not like any known interacting binary. As time passes and the orbit decays, the secondary may eventually overflow its Roche lobe and turn V471 Tau into an intermediate polar, but for now it remains in a class by itself.

We are grateful for support from NSERC Canada, CNRS France, SERC UK, CNPQ Brazil, the National Science Foun- dation grants AST85-52456, AST86-00507, AST87-12249, AST88-13572, NASA grant NGT-50210, and grant 3547-87 from the National Geographic Society.

We acknowledge use of the EXOSAT archives at ESTEC and HEASARC.

J. C. C. also thanks Bill Jefferys and the other authors of the program Gaussfit and acknowledges W. Disney for providing an accurate clock which was used to verify some of the timings.

\section{APPENDIX}

The reprocessing time is a combination of the diffusion time of the ionizing photons into the chromosphere, the diffusion time of the escaping Balmer continuum photons and the time required for fluorescent reprocessing. Detailed photon diffusion calculations are well beyond the scope of this paper. We argue that the diffusion time of the incoming ionizing photons is much shorter than 555 s, since for these wavelengths scattering is negligible and absorption occurs, by definition, at the reprocessing site. Likewise, the diffusion time for the escaping photons is short since above the reprocessing region the chromosphere is optically thin at Balmer continuum wavelengths. Thus we ignore the contribution of photon diffusion to the reprocessing time.

The time scale for fluorescent reprocessing is the time it takes for the atoms which were ionized by the incident flux to recombine once the flux is decreased. From Osterbrock (1974), this recombination time scale for an optically thick region is

$$
\tau_{r}=\frac{1}{N_{e} \alpha_{B}}
$$

where $N_{e}$ is the electron density and $\alpha_{B}$ is the recombination coefficient for the hydrogen atom summed over all levels above ground level.

We can find $N_{e}$ at the site of reprocessing from the measurements of Young et al. (1988). At orbital phase 0.5, they find an excess of $1.3 \times 10^{29} \mathrm{ergs} \mathrm{s}^{-1} \mathrm{H} \alpha$ emission over the level at orbital phase 0.0. This represents an excess of $4.3 \times 10^{40}$ photons $\mathrm{s}^{-1}$. The emission rate of $\mathrm{H} \alpha$ photons per unit volume can be expressed as

$$
J=N_{e} N_{p} \alpha_{\mathrm{H} \alpha}^{\mathrm{eff}},
$$

where $N_{p}$ is the proton density and $\alpha_{\mathrm{H} \alpha}^{\text {eff }}$ is the effective recombination rate for electrons which result in H $\alpha$ photons. If we assume complete ionization of hydrogen in the reprocessing region, then $N_{e} N_{p}=N_{e}^{2}$. Now we multiply the equation above by volume $V$. 
The left-hand side will be $J V$, which is the total excess $\mathrm{H} \alpha$ generated on the illuminated side of the $\mathrm{K}$ star. We calculated this number above. Furthermore, we can estimate a value of $V$ to substitute into the right-hand side. We take it to be the product of one chromospheric scale height and half the surface area of the $\mathrm{K}$ star. We have used $10^{7} \mathrm{~cm}$ for the scale height, $5 \times 10^{10} \mathrm{~cm}$ for the on' radius of the K star, and $1.6 \times 10^{-13} \mathrm{~cm}^{3} \mathrm{~s}^{-1}$ for $\alpha_{\mathrm{H} \alpha}^{\text {eff }}$ (Osterbrock 1974). Substituting yields $N_{e}=1.25 \times 10^{12} \mathrm{~cm}^{-3}$.

Ind This number, with $\alpha_{B}$ from Osterbrock gives a recombination time scale of $\tau_{r} \sim 1 \mathrm{~s}$.

\section{REFERENCES}

Bois, B., Lanning, H. H., \& Mochnacki, S. W. 1988, AJ, 96, 157

Illarionov, A. F., \& Sunyaev, R. A. 1975, A\&A, 39, 185 Jensen, K. A., Swank, J. H., Petre, R., Guinan, E. F., Sion, E. M., \& Shipman,
H. L. 1986, ApJ, 309, L27

Kepler, S. O., Robinson, E. L., Nather, R. E., \& McGraw, J. T. 1982, ApJ, 254, 676

Nather, R. E., Winget, D. E., Clemens, J. C., Hansen, C. J., \& Hine, B. P. 1990, ApJ, 361, 309

Nelson, B., \& Young, A. 1970, PASP, 82, 699

Osterbrock, D. E. 1974, Astrophysics of Gaseous Nebulae (San Francisco: Freeman)

Patterson, J., \& Price, C. M. 1981, ApJ, 243, L83

Robinson, E. L., Clemens, J. C., \& Hine, B. P. 1988, ApJ, 331, L29

Robinson, E. L., Kepler, S. O., \& Nather, R. E. 1982, ApJ, 259, 219

Skillman, D. R., \& Patterson, J. 1988, AJ, 96, 976

Winget, D. E. 1981, Ph.D. thesis, University of Rochester

Winget, D. E. \& Claver, C. F. 1989, in IAU Colloq. 114, White Dwarfs, ed. G. Wegner (Berlin: Springer), 293

Winget, D. E., Nather, R. E., \& Hill, J. A. 1987, ApJ, 316, 305

Young, A., \& Skumanich, A. 1983, BAAS, 15, 917

Young, A., Skumanich, A., \& Paylor, V. 1988, ApJ, 334, 397 